\title{
BRITISH MEDICAL ASSOCIATION
}

\author{
ANNUAL MEETING, 1950
}

In Liverpool on July 20, 1950, the Section of Dermatology devoted a morning session to Venereal Diseases. This was the first occasion for many years that the specialty of venereology has occupied an official place in the Annual Meetings of the B.M.A. The chemotherapy of venereal diseases was a pertinent subject which produced much valuable discussion and the two opening papers are published below by permission of the Editor of the BRITISH MEDICAL JOURNAL.

\section{CHEMOTHERAPY OF VENEREAL DISEASES ITS USES AND ABUSES

\author{
BY \\ ROBERT LEES
}

Manchester

The history of venereal disease has many examples of the development and abuse of chemotherapy. There have been many conspicuous discoveries, and high hopes have been raised, but misuse and abuse and the reaction against extravagant claims have led to disappointment and the neglect of useful methods.

Mercury was used by the Chinese in the treatment of cutaneous disease from about 500 B.C., and it has been in common use in Arabian therapeutics for many centuries, but when mercury was adopted into European medical practice it was greatly misused and fell into disrepute. The physicians and quacks who professed to cure skin diseases and the "great pox", caused horrid suffering or even the death of some of their patients. The old medical works contain lurid descriptions of the salivations, mouth ulceration, foetor, and cachexia often caused by mercury.

The Unguentum Saracenicum, brought back from the Crusades to Europe, contained mercury, and was found very effective in many of the parasitic and luetic skin diseases of that age. Its action was probably a remote chemo-therapeutic action rather than a direct antiseptic action, as it was applied to healthy skin. But the Unguentum Saracenicum too was so misused that it fell into disrepute, and the practitioners shared some of its loss of reputation.

In more recent years we have had similar high hopes aroused by the advent of iodides, salvarsan, bismuth, sulphonamides and penicillin. It has been our boast that we were the principal practitioners of "specific treatment"; that exact laboratory methods were available not only for accurate diagnosis before treatment but also for the scientific control of the chemotherapy. Often, however, these methods were not as accurate as their practitioners believed, for the biology of the infecting organisms is not fully known. Exaggerated claims have been made ; these were usually due to excessive enthusiasm, but also to the lack of a scientific spirit of observation and critical evaluation. On the whole, however, in the last forty years the treatment of syphilis has been an example of precise diagnosis, and rational chemotherapy.

At present there is again a tendency to depart from these standards, and to give haphazard and empirical treatment, offering the excuse that if it does not do good at least it will not do harm. So the title of this paper is two-headed, and we will discuss the abuses as well as the uses of chemotherapy.

Since most of us are engaged in practical treatment rather than scientific investigation, $I$ think it will be preferable to present the subject under the headings of the common manifestations of disease rather than to approach it from the accurate experimental side and compare that with current practice.

\section{Gonorrhoea}

This aspect will interest the venereologists more than the dermatologists, most of whom have 
given up the attempt to practise two specialties. The most effective remedy in common use is penicillin, and the aqueous suspensions of penicillin procaine provide an almost ideal remedy. The dosage required is about 300,000 or 400,000 units ; $1 \mathrm{ml}$. of the usual suspensions given by intramuscular injection will maintain an effective tissue concentration for 24 hours.

Apparent recovery follows in about 95 per cent. of cases, while the remaining 5 per cent. have a period of benefit followed by speedy relapse in which gonococci may be readily detected in the secretions of the genito-urinary tract. It has been claimed, however, that a high proportion of the apparent "cures" are not cured but merely symptom free. If additional treatment is not given, these persons either have an obvious clinical and bacteriological relapse after a few weeks or months, or they may develop chronic disease or become carriers. It is therefore essential to employ accurate methods and to apply stringent standards to establish cure.

Giving a much higher dosage of penicillin does not altogether overcome this difficulty. Cases have been recorded where gonococci have been found in patients who had just received over 2 mega units penicillin as treatment for syphilis.

An objection to high doses of penicillin is the possibility of concealing and overlooking concomitant syphilitic infection. It is probable that the best results are obtained by the simultaneous use of sulphonamides and penicillin, together with repeated tests at short intervals, always using a reliable technique of culture, with retreatment of failures.

The simultaneous use of penicillin and sulphonamides may have a synergic action on gonorrhoea; it is also an effective attack on Gram positive organisms which often cause persistent urethritis. In practice, too, it affords an invaluable opportunity to keep the patient coming for treatment; this is not to provide us with more work, but to give a better chance of securing efficient social work to trace the source of the disease and gain the patient's cooperation in establishing cure. I do not hesitate at times to use harmless dyes as a placebo, and to stimulate the patient's interest in cure. It is not difficult to produce urine ranging in colour from azure blue to sea green, deep yellow, or rose red.

Streptomycin is extremely effective in gonorrhoea, and if cost is not counted, it is preferable to penicillin, as it has very little effect on syphilis and a marked effect on many of the organisms which cause urethritis. Aureomycin and other antibiotics are not yet practical remedies as cost precludes their use.
Abuses.-The commonest abuses in the chemotherapy of gonorrhoea are :

(i) treatment without accurate diagnosis,

(ii) treatment without efficient test of cure,

(iii) over-dosage with penicillin,

(iv) neglect of ancillary treatment,

(v) reckless use of antibiotics for the prevention of infection.

(i) The dangers of treatment without diagnosis are that legal difficulties may arise, matrimonial disputes being a not uncommon sequel to an ill-founded diagnosis of gonorrhoea. To-day it is probably true that only about 50 per cent. of cases of urethral discharge are gonococcal. Accurate diagnosis is almost impossible if treatment precedes diagnosis; this is serious if relapse occurs or complications such as arthritis or salpingitis develop.

(ii) The danger of inefficient tests of cure is evident mainly to venereologists who are perturbed at the possibility of failure to reduce the incidence of gonorrhoea in the population, and the persistence of postgonorrhoeal disease such as pelvic infections of women and chronic prostatic infections in men.

(iii) It is a commonplace to see patients who have had gonorrhoea treated by a practitioner who has given daily injections of penicillin for as long as a week. The objections to this are the waste of a valuable drug, the risk of concealment of incubating syphilis, and the subjection of the patient to unnecessary injections. I can think of no excuse for this over-dosage, and the only reason I can find for it is the desire of unscrupulous persons to charge heavier fees to a patient who is not likely to make public complaint.

(iv) Neglect of ancillary treatment is most common in females, many of whom have co-incident disease due to trichomonads or monilia ; but warts in both sexes, and prostatitis in male patients are often neglected. It is also common for a too mechanical or a too physical view of the patient to be adopted, and the psychological factors to be ignored. It must be recognized that every venereal patient has special mental problems which have produced promiscuous sexual conduct, or arise from the disease thereby acquired. He is not cured unless the mental factors of his illness have had due attention.

(v) The use of penicillin and other chemo-therapeutic agents for the prophylaxis of disease may have been pardonable as an expedient during war, but have little place in present life. The objections are firstly moral, in that the possession of an apparently effective and safe drug as a guarantee against contracting venereal disease leads to more general sexual promiscuity and consequent weakening of the social structure of life. Secondly, and I think more important, is the certainty that drugresistance will be induced, so that the therapeutic use of penicillin for staphylococcal and streptococcal infection will be threatened.

It is not improbable that in many persons syphilis may develop without any visible sign until the destructive lesions of late syphilis become obvious. 


\section{Syphilis}

Early Syphilis.-It is difficult to be dogmatic on this subject, since a considerable period of time is essential to test any remedy for syphilis. The initial dosage scheme of 2.4 mega units in $7 \frac{1}{2}$ days which was used in the services in the war has had a high relapse rate. I have found, in civilian practice, that about 5 per cent. of such patients still suffer from syphilis and have no suspicion that penicillin has failed to cure them. This is found, for example, when they request a test prior to marriage, or if they are examined for recently acquired gonorrhoea. The reaction to "too little" has probably been " too much", and current dosage schedules may err on the side of over-dosage. We have been unduly concerned with speedy treatment of syphilis, and try to condense our schedules of injections into a very short time. In part this is forced upon us by the tendency of the patient to disappear from the surgery or clinic as soon as the obvious signs of disease fade. The optimum amount of penicillin for early syphilis is not known, but a high cure rate probably results from amounts between 5 and 10 mega units given in 7 to 10 days. I do not think either benefit or harm results from higher doses, but extension of the time of penicillin treatment to 20 days might be beneficial, and this is now less likely to meet with objections from the patient as the newer preparations are painless and less likely to produce urticarial reactions.

We do not know if penicillin alone can give a one hundred per cent. cure rate in early syphilis. It is probable that this is true, but the necessary dosage scheme is unknown. Treatment schemes which combine the use of penicillin and bismuth, and others which use penicillin, bismuth, and arsphenamine products are in common use. The penicillinbismuth scheme is safe, and time will prove whether it has a very high cure rate. The principal reason for neglect to use the arsenical drugs in the treatment of syphilis is fear of their toxic action, though some physicians hold that they are no longer necessary. It is our belief that simple care and precautions can eliminate most of the risks attending the use of arsenicals, and it is probable that their use may make the 100 per cent. cure rate more certain.

We advocate a schedule in which all three drugs are used simultaneously in an early intensive attack on the infection, and this is then followed up by a long period of much less intensive chemotherapeutic attack. The intensive attack is designed to last 10 to 14 days and is based on the probable synergic action of these three groups of drug. It produces a very rapid control of surface contagion. If the patient defaults, as many do whatever system is used, it is probable that the majority are either cured or remain non-contagious for life, provided they have completed the programme of treatment for the first three weeks. The patient can be treated on an ambulatory basis, though I do not hesitate to admit to hospital those who may be a public danger or who are unlikely to complete their intensive course. Obvious examples of this class are seamen, transport drivers, prostitutes, and theatrical artistes. We have not found there is any great risk of Herxheimer reactions or other toxic effects from this triple attack, but this intensive treatment should not be given to pregnant women; this aspect of the subject is discussed below.

The arsenical drug is given along with bismuth on the first day of treatment and the arsenical is then given once or twice per week according to the preparation used. Penicillin injections start on the second day, and are given daily for 10 to 16 days, the usual dose being 600,000 units of penicillin procaine each day. Higher dosage, such as 900,000 units daily, produces more pain and as the blood and tissue level is not much higher there is little advantage in increasing the daily dose. There seems to be little place in the treatment of early syphilis for giving aqueous solution of penicillin every 3 hours, though I have employed (e.g. in pregnancy) the combination of 3-hourly injection of aqueous solution during the day (to produce very high blood levels) with a bed-time dose of penicillin procaine to ensure continuous penicillin action.

The results in early syphilis appear to be good. The surface lesions heal with great speed, the patient becomes non-contagious very rapidly, and the symptoms and signs are rapidly relieved. It is too early to claim final results and nothing less than 100 per cent. permanent cure, with no mortality, and a negligible incidence of toxic effects, should be accepted.

In my experience the incidence of allergic reactions is low, and respond well to anti-histamine drugs, particularly if the antidote is used early. The incidence of serious arsenical reactions varies greatly and is in indirect proportion to the care taken in observing and testing the patient before each treatment. I have found BAL to be a rapid and almost certain antidote to cutaneous reactions such as erythema or arsenical dermatitis, provided it is used in effective doses within 24 hours of the appearance of the skin erythema. If the administration of BAL is delayed for several days (two or more) the results are likely to be disappointing. I now regard sodium thiosulphate, however administered, to be quite valueless in arsphenamine toxic reactions.

The toxic reaction to be feared is arsenical encephalopathy, and this can be avoided, except in 
the rarest instances of idiosyncrasy, by avoiding intensive dosage with arsenical drugs. There is an additional susceptibility to this complication during pregnancy and accordingly I now omit all arsenical drugs from the treatment of a pregnant woman.

Late Syphilis.-The place of antibiotics in the treatment of late syphilis is still being explored, but we know that penicillin has great value in neurosyphilis, even though the concentration of the drug in the central nervous system and cerebrospinal fluid is very low. Intrathecal treatment is very rarely required or beneficial. In gummatous conditions, whether superficial and affecting the skin and connective tissues or visceral, penicillin can replace the arsenical compounds with equal therapeutic effect and much greater safety. It is probably necessary to give repeated " courses" of injections, each course lasting about 10 to 14 days, separated by intervals of 6 months.

Penicillin should be supplemented by courses of bismuth and iodides in such cases.

Cardiovascular Syphilis.-The precise place of penicillin in the treatment of this condition is still unknown, but I suggest that it can replace the arsenical drugs, and that the same care should attend its administration. There is less fear of serious Herxheimer reactions, but there is equally great danger of a " therapeutic paradox ", i.e. rapid improvement followed by a rapid and permanent deterioration which is ascribed to excessive fibrosis during healing. I have seen several patients who have been treated by penicillin for neurological symptoms with conspicuous improvement, but who developed rapidly progressive cardiac failure within 6 months. I was convinced that this was a " therapeutic paradox", and I am sure that recognition of asymptomatic cardiovascular disease, and more careful preparation of the patient with iodides and bismuth, would have prevented this irreversible process.

Congenital Syphilis.-It is difficult to be sure what chemotherapy achieves in this condition, as the clinical course is so variable and it is difficult to separate active luetic processes from allergic manifestations. In early childhood penicillin is dramatically curative.
In cases of interstitial keratitis benefit is not seen unless a special technique is adopted to ensure a high concentration of penicillin in the eye. This means very high dosage and often sub-conjunctival injections of penicillin.

The possibility of preventing congenital syphilis is now so easily within our reach that there should not be a single congenital syphilitic baby in Britain by 1970 . Penicillin given to the pregnant woman in adequate amounts between the 4th and 7th month of pregnancy will prevent congenital syphilis in about 99 per cent. of instances. If the penicillin course is supplemented by a bismuth injection each week up to the last month of pregnancy I am sure that congenital syphilis will always be prevented.

Abuses.-What are the abuses of chemotherapy in syphilis? Here again they are the use of "too little", or " too much". I have known patients endure weekly injections of heavy metals for as long as ten years, just because the blood gave a positive Wassermann reaction in some degree. The only result I expect from such therapy is chronic metallic poisoning and drug-fastness. At the opposite extreme I have known a glib practitioner give a few injections of penicillin " just in case there might be a touch of syphilis ", leaving the patient a prey to secret fears, and all other doctors uncertain whether the patient had ever suffered from syphilis or not.

The local application of penicillin and other substances of similar type has no place in the rational treatment of syphilis. At the best it does no good and no harm, but as a rule it confuses the diagnosis.

In a different category is the treatment of the healthy with large amounts of chemotherapy, and the commonest instances I have seen are the administration of anti-syphilitic treatment :

(i) to the healthy children of women who formerly suffered from syphilis.

(ii) to men with non-syphilitic lesions of the genitals such as chancroid, warts, epithelioma, and even herpes.

(iii) to patients with leucoplakia, psoriasis, lichen planus, and some others of the common imitators of syphilis. 